$10-2000$

\title{
Classically Forbidden Recurrences in the Photoabsorption Spectrum of Lithium
}

\author{
V. Kondratovich \\ William \& Mary \\ John B. Delos \\ William \& Mary, jbdelos@wm.edu \\ Neal Spellmeyer \\ Daniel Kleppner
}

Follow this and additional works at: https://scholarworks.wm.edu/aspubs

Part of the Physics Commons

\section{Recommended Citation}

Kondratovich, V.; Delos, John B.; Spellmeyer, Neal; and Kleppner, Daniel, Classically Forbidden Recurrences in the Photoabsorption Spectrum of Lithium (2000). Physical Review A, 62(4).

https://doi.org/10.1103/PhysRevA.62.043409

This Article is brought to you for free and open access by the Arts and Sciences at W\&M ScholarWorks. It has been accepted for inclusion in Arts \& Sciences Articles by an authorized administrator of W\&M ScholarWorks. For more information, please contact scholarworks@wm.edu. 


\title{
Classically forbidden recurrences in the photoabsorption spectrum of lithium
}

\author{
Vladimir Kondratovich and John B. Delos \\ Department of Physics, College of William and Mary, Williamsburg, Virginia 23187 \\ Neal Spellmeyer* and Daniel Kleppner \\ Research Laboratory of Electronics, George R. Harrison Spectroscopy Laboratory and Department of Physics, \\ Massachusetts Institute of Technology, 77 Massachusetts Avenue, Cambridge, Massachusetts 02139
}

(Received 9 May 2000; published 19 September 2000)

\begin{abstract}
We present data on the photoabsorption spectrum of lithium atoms in an electric field at energies between the saddle point of the Stark potential and below the field-free ionization threshold. The spectrum displays a sequence of sharp resonances and a sequence of broad ones. We find that the broad resonances arise from the classically forbidden reflection of waves above a dynamical potential barrier. The recurrence spectrum is also observed and it is dramatically affected by above-barrier reflections. We have developed a semiclassical theory that interprets the spectra using quasiclassical trajectories that undergo above-barrier reflection.
\end{abstract}

PACS number(s): 32.60.+i, 03.65.Sq, 32.30.-r, 32.80.-t

In this paper, we identify resonances in an atomic absorption spectrum that are associated with a classically forbidden process: reflection of a quantum wave when the effective energy is above the top of a potential-energy barrier (Figs. 1-3) [1]. In this circumstance, a classical particle would directly escape from the atom. The corresponding quantum wave, however, has a reflection coefficient. We identify here broad resonances associated with this "above-barrier reflection."

In our analysis, we provide a quantitative description of the spectrum by a new method. Also we extend closed-orbit theory [2] by introducing quasiclassical closed orbits undergoing above-barrier reflection.

\section{CLASSICALLY ALLOWED AND CLASSICALLY FORBIDDEN REFLECTIONS IN A HYDROGEN ATOM IN AN ELECTRIC FIELD}

Several phenomena in atomic spectroscopy manifest themselves as broad structures in the absorption spectrum, and these can easily be confused with above-barrier reflection. (A) If a negative ion is placed in an electric field, a part of the wave function of the photodetached electron travels uphill against the electric force, and then is reflected back downhill. The returning wave overlaps the atom and interferes with the outgoing wave, producing oscillations in the absorption spectrum [3]. (B) If a neutral atom is placed in an electric field, and the absorption spectrum is measured above the field-free threshold, waves that travel uphill are also reflected back to the atom, again giving interference modulations or resonances [4]. Similar phenomena occur in a magnetic field [5]. These phenomena involve classically allowed reflection (i.e., classically required reflection): the wave follows the path that would be followed by a classical particle of the same initial momentum, and the reflection coefficient is unity.

\footnotetext{
*Present address: MIT Lincoln Laboratory, Lexington, MA 02420 .
}

For an atom in an electric field below the field-free ionization threshold, the situation is more complex. Let us restrict ourselves to the hydrogen atom in an electric field.

The potential energy of the electron

$$
V(\mathbf{r})=-1 / r+F z
$$

has a saddle at energy $E_{s}=-2 F^{1 / 2}$. Classically, if an electron leaves the atom with an energy below $E_{s}$, then no matter what direction it goes, it is reflected from a potentialenergy hill, and it remains bound to the atom forever. In quantum theory, such an electron escapes from the atom only by tunneling. Accordingly, the absorption spectrum consists of a set of narrow resonances, with a lifetime governed by the rate of tunneling through the barrier (or the rate of spontaneous emission of a photon, whichever is larger).

If the electron is ejected from the atom with an energy between the saddle energy and the zero-field ionization energy $E_{s}<E<0$, then its fate is determined by its initial direction of motion. The electron has enough energy to escape, but unless it has sufficient momentum in the "downhill" direction, it will still remain bound to the atom. There is a critical angle $\theta_{c}$, such that electrons leaving the atom with $\theta_{c}<\theta \leqslant \pi$ escape quickly, but those leaving the atom with $0 \leqslant \theta<\theta_{c}$ are bound forever. (Those leaving at $\theta_{c}$ approach a periodic orbit that lies on a parabola not far from the potential-energy saddle.)

The critical angle $\theta_{c}$ depends on the energy as

$$
\cos \theta_{c}=1-E^{2} / 2 F
$$

so as the energy is raised from $E_{s}$ to zero, the critical angle varies between $\pi$ and 0 : the bound sector shrinks and the escape sector grows.

What are the consequences for the quantum spectrum in this energy range? Trajectories in the bound sector again correspond to long-lived quantum states; their lifetimes are governed not by tunneling through the real potential-energy barrier (Fig. 1) but by tunneling through the effective potential-energy barrier (Fig. 2). These long-lived states appear in the absorption spectrum as narrow resonances. In 


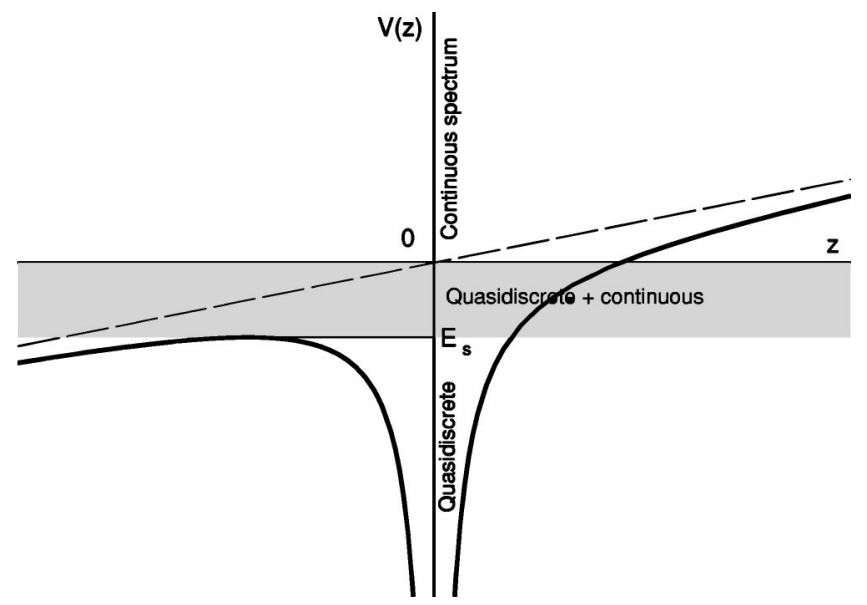

FIG. 1. The Stark potential energy [Eq. (1.1)] has a potential energy barrier, below which electrons can escape only by tunneling. Above the saddle-energy $E_{s}$, electrons have enough energy to escape, but they may still be classically bound forever by a dynamical barrier (Fig. 2). Above the dynamical barrier they may still be bound temporarily by classically-forbidden above-barrier reflection.

contrast, quantum states associated with the classical escape sector are essentially free states, and they give continuum absorption. It follows that the absorption spectrum as a function of energy consists of narrow lines superposed on a smoothly rising continuum (see Fig. 4 of Ref. [6]).

Interesting phenomena occur if the initial direction of the electron is close to the critical angle. While the trajectories change discontinuously from bound to free if the ejection angle $\theta$ is increased through $\theta_{c}$, the corresponding quantum states must be continuously connected. States having electron ejection angles slightly on the bound side of $\theta_{c}$ have relatively short tunneling lifetimes (compared to other quasibound states), and therefore they appear as relatively broad resonances. States having electron ejection angles slightly on the free side of $\theta_{c}$ have relatively long lifetimes (compared to other continuum states), and therefore they appear as "structure in the continuum.' If we were to vary the electric field such that the ejection angle associated with a particular quasibound resonance were to increase through $\theta_{c}$, we would see the width of the resonance increase rapidly, so its
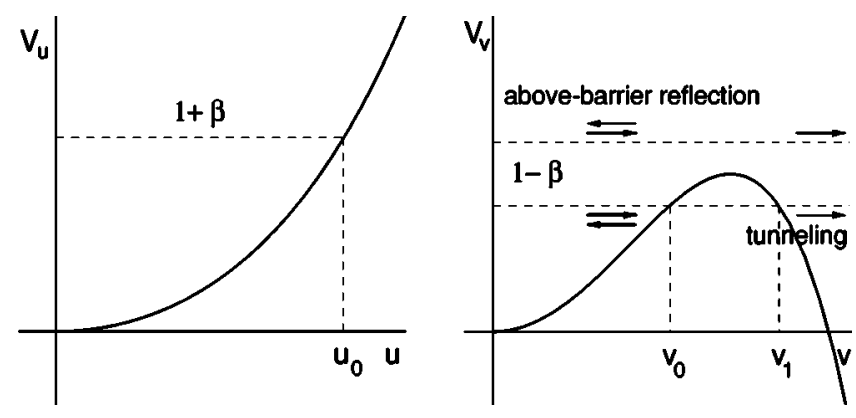

FIG. 2. Effective potentials for motion along $u$ and $v$ coordinates. The quantities $1+\beta$ and $1-\beta$ play the role of effective energies. The $u$ motion is always bound; the $v$ motion can show tunneling or above-barrier reflections, both of which are classically forbidden.

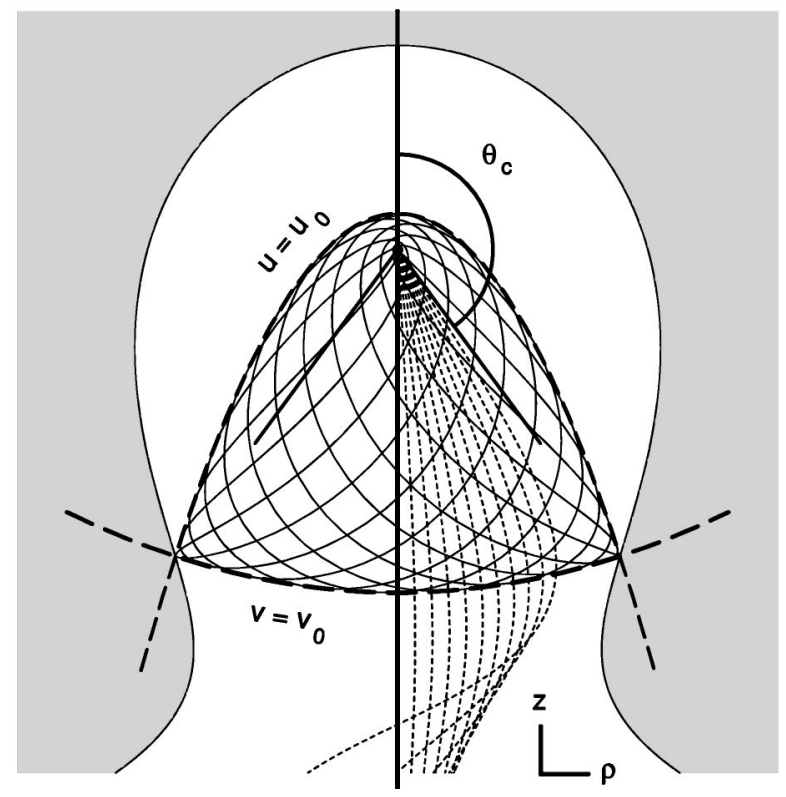

FIG. 3. Bound trajectory (solid line) and escaping trajectories (dashed lines) in Coulomb field combined with electric field along the $z$ axis. The scaled energy, $\varepsilon=-1.9$, is slightly above the saddle point energy, $\varepsilon_{\text {saddle }}=-2$. All trajectories have enough energy to escape, but those having ejection angle $\theta \leqslant \theta_{c}$ are bound by the dynamical barrier in the $v$ coordinate. The energetically forbidden region is shaded.

contribution to the absorption spectrum would change from a narrow line to a smooth contribution to the continuum.

When the ejection angle is just on the free side of $\theta_{c}$, the lifetime of the state is governed by the classically forbidden process of above-barrier reflection. In this paper, we identify in the Stark absorption spectrum of Li a set of broad resonances that correspond to classically forbidden above-barrier reflection. The same region of the spectrum has been examined in a number of studies [7], but none have previously noted this particular phenomenon. It is of special interest to us because this phenomenon forces us to give a new extension of closed-orbit theory, introducing quasiclassical closed orbits undergoing above-barrier reflection.

\section{HYDROGENIC STARK PROBLEM}

The Schrödinger equation and the classical HamiltonJacobi equation are separable in parabolic coordinates $u$ $=\sqrt{r+z}, \quad v=\sqrt{r-z}$, with effective Hamiltonians

$$
\begin{aligned}
& h_{u}=p_{u}^{2} / 2-E u^{2}+F u^{4} / 2=1+\beta, \\
& h_{v}=p_{v}^{2} / 2-E v^{2}-F v^{4} / 2=1-\beta .
\end{aligned}
$$

Everything we said in Sec. I is easily proved by analysis of these equations. The separation constant $\beta(-1 \leqslant \beta \leqslant 1)$ is related to the polar angle $\theta$ at which a classical electron leaves the atom by

$$
\beta=\cos \theta
$$


Also $1 \pm \beta$ plays the role of the effective energy associated with the $u$ or $v$ motions, respectively (Fig. 2).

The motion along the $u$ coordinate is always bound (Figs. 2 and 3). However, in the $v$ coordinate, there is an "effective" or "dynamical" potential-energy barrier. If an electron with energy above $E_{s}$ leaves the atom in a "downhill" direction, then $\cos \theta \sim-1, h_{v}=1-\beta$ is large, and the electron can escape over the barrier. If the electron leaves the atom in an uphill direction, then $\cos \theta \sim 1, h_{v}=1-\beta$ is small, and the electron is trapped by the dynamical barrier. It has enough energy to escape (Fig. 1), but it is trapped below the dynamical barrier (Fig. 2), i.e., it never finds the escape route (Fig. 3).

It also follows easily from Eqs. (2.1) that the boundary between bound and free motions is at $\beta_{c}=\cos \theta_{c}=1$ $-E^{2} / 2 F$. If the electron leaves the atom just slightly downhill from this critical angle then the value of $h_{v}=1-\beta$ is just barely higher than the dynamical barrier. A classical particle moves slowly over the barrier and escapes, but the quantum wave is partially reflected and partially transmitted. This classically forbidden above-barrier reflection produces broad resonances in the absorption spectrum.

\section{OBSERVATIONS AND INTERPRETATION BY WKB APPROXIMATION}

Scaled energy spectroscopy [8(a)] is invaluable for observing these states experimentally. In this method the excitation energy $E$ and electric field $F$ are simultaneously varied so that the scaled energy $\varepsilon=E / F^{1 / 2}$ is constant. The spectrum is recorded as the scaling variable $w=F^{-1 / 4}$ is varied. Scaled energy spectroscopy maintains constant the shapes of classical orbits as it generates the absorption spectrum $\left.D f(E, F)\right|_{\varepsilon=\text { const }} \equiv D f(w)$.

The experimental setup is similar to that described in [8(b)]. Lithium is excited to the $3 s$ state by two-step resonant excitation $2 s \rightarrow 2 p \rightarrow 3 s$, and then to an $m=0$ Rydberg state by a tunable laser. The absorption spectrum, Fig. 4, was taken for scaled energy $\varepsilon=E / \sqrt{F}=-0.125$. This is far above the Stark saddle energy $\varepsilon_{\text {saddle }}=-2$ and the critical angle is about $7^{\circ}$ from the uphill electric field axis.

We see in the absorption spectrum a reasonably flat continuum, a sequence of four narrow resonances, and a sequence of four less intense and broader resonances $\left(n_{v}=1\right)$. These are associated, respectively, with escaping classical orbits, bound classical orbits, and quasiclassical orbits that undergo quantum above-barrier reflection.

To make this connection, we extend semiclassical quantization conditions above the dynamical potential barrier by using a uniform-WKB approximation (see, for example, [9]). This leads to complex quantization conditions

$$
\begin{gathered}
J_{u}(E, \beta)=\left(n_{u}+1 / 2\right) 2 \pi, \\
J_{v}(E, \beta)=\left(n_{v}+1 / 2\right) 2 \pi-i \lambda_{v}-\delta,
\end{gathered}
$$

where $J_{u}$ and $J_{v}$ are action variables associated with the $u$ and $v$ motion

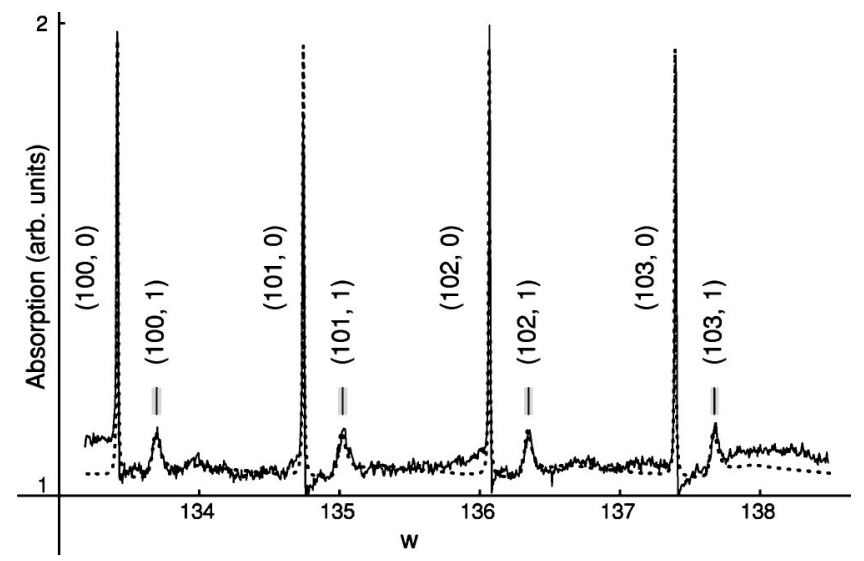

FIG. 4. Scaled absorption spectrum of Li vs $w=F^{-1 / 4}$ at $\varepsilon$ $=-0.125$, experiment and theory (dotted line). The parabolic quantum numbers $\left(n_{u}, n_{v}\right)$ are indicated. All peaks correspond to resonances that are far above the potential-energy saddle. States with $n_{v}=0$ are below the dynamical barrier; escape is classically forbidden and they decay by tunneling. Those with $n_{v}=1$ are above the dynamical barrier and escape is classically allowed; they have a lifetime because of quantum reflection above the dynamical barrier. The shadowed strips show the position and widths of these states.

$$
\begin{aligned}
& J_{u}(E, \beta)=\int_{o s c} p_{u} d u=2 \int_{0}^{u_{0}} \sqrt{2(1+\beta)+2 E u^{2}-F u^{4}} d u, \\
& J_{v}(E, \beta)=\int_{o s c} p_{v} d v=2 \int_{0}^{v_{0}} \sqrt{2(1-\beta)+2 E v^{2}+F v^{4}} d u,
\end{aligned}
$$

$\lambda_{v}$ describes the imaginary addend to action due to tunneling or above-barrier reflection

$$
\lambda_{v}=\frac{1}{2} \ln \left(1+e^{-2 K}\right)
$$

and $K$ is an underbarrier action integral

$$
K=\int_{v_{0}}^{v_{1}} p_{v} d v
$$

The cuts on the complex $v$ plane are defined such that $K$ is positive below the barrier and negative above the barrier. $\sigma$ is a parabolic-barrier phase correction

$$
\delta=\arg \Gamma\left[\frac{1}{2}+i \frac{K}{2 \pi}\right]-\frac{K}{2 \pi} \ln \left(\frac{|K|}{2 \pi}\right)+\frac{K}{2 \pi} .
$$

The quantization conditions (3.1) are associated with complex turning points, and they lead to complex quantized values of $E$ and $\beta$.

Using these quantization conditions, we identify the narrow peaks in the experimental spectrum (Fig. 4) as the levels with parabolic quantum numbers $n_{u}=100-103$ and $n_{v}=0$. These are ordinary above-saddle-energy Stark states. Each of these states has an energy above the saddle in $V(\mathbf{r})$, but its effective energy in the $v$ coordinate $1-\beta$ is below the bar- 
rier in the effective potential energy $V_{v}$ (Fig. 2), so reflection is classically mandated, and the state has a long lifetime.

By the same calculation we identify the broad peaks in Fig. 4 as above-barrier levels with $n_{v}=1$. These arise from classically forbidden reflection above the effective potentialenergy barrier.

\section{SEMIQUANTAL CALCULATION OF THE ABSORPTION SPECTRUM}

We have computed the absorption spectrum including continuum, quasidiscrete states and above-barrier reflection using a new semiclassical method. Details of the theory will be given in a later paper; here we give only a sketch of the essential results.

If we have only a discrete spectrum, then the oscillator strength for the transition from a given initial state $\mathbf{i}$ to a final state $\mathbf{n}$ is defined as

$$
f_{\mathbf{n}}^{\mathbf{i}}=2\left(E_{\mathbf{n}}-E_{\mathbf{i}}\right)|\langle\mathbf{i}|D| \mathbf{n}\rangle|^{2},
$$

where $D$ is the relevant component of the dipole operator. The oscillator-strength-density $D f(E)$ is defined as

$$
D f(E)=\sum_{\mathbf{n}} f_{\mathbf{n}}^{\mathbf{i}} \delta\left(E-E_{\mathbf{n}}\right) .
$$

In a previous paper [10], we presented a semiclassical formula for the oscillator strength of discrete high-Rydberg states,

$$
f_{\mathbf{n}}^{\mathbf{i}}=\left.32 \pi^{3}\left(E_{\mathbf{n}}-E_{\mathbf{i}}\right)\left|\frac{\partial(E, \beta)}{\partial\left(J_{u}, J_{v}\right)}\right| \mathcal{Y}\left(\theta_{\mathbf{n}}\right)\right|^{2} .
$$

$\mathcal{Y}(\theta)$ is the angular distribution of electron waves going out from the atom as a result of excitation by light.

In another paper [11], following the method of Berry and Tabor [12], we used the Poisson sum formula to transform the oscillator-strength density $D f(E)(4.2)$ and (4.3) to the form

$$
D f(E)=32 \pi^{3}\left(E-E_{\mathbf{i}}\right) \sum_{\mathbf{M}} \int d \beta e^{i \mathbf{M}(\mathbf{J}-\boldsymbol{\mu} \pi / 2)}|\mathcal{Y}(\theta(E, \beta))|^{2} .
$$

Here the integral over the conserved quantity $\beta$ is an integral over all possible tori that exist at the given energy $E$. The sum over all integers $\mathbf{M}$ is a sum over all possible loops on those tori. $M_{u} J_{u}+M_{v} J_{v}$ is the action integral around those loops, and we include in the sum $\left(-\infty<M_{u}, M_{v}\right.$ $<\infty) . \mu$ is the vector of Maslov indices for single loops on the tori; in our case $\boldsymbol{\mu}=(2,2)$.

As stated above, Eq. (4.4) applies to a discrete spectrum, when there is no tunneling. In the present case, we have tunneling in the $v$ coordinate, and Eq. (4.4) must be modified. A lengthy analysis shows that a very similar representation holds when tunneling is present and the semiclassical quantization conditions are complex. Let us define

$$
\mathcal{R}_{v}(E, \beta)=e^{-\lambda_{v}}=\left(1+e^{-2 K(E, \beta)}\right)^{-1 / 2}
$$

as the reflection coefficient for each cycle of $v$ motion. [ $K$ is the underbarrier integral defined in Eq. (3.4).] The analysis (which we will present elsewhere) shows that the exponential factor $\exp \left(i M_{v} J_{v}\right)$ in Eq. (4.4) should be multiplied by the reflection coefficient $\mathcal{R}_{v}$ once per cycle, to give a factor $\mathcal{R}_{v}^{\left|M_{v}\right|}$ :

$$
\begin{aligned}
D f(E)= & 32 \pi^{3}\left(E-E_{\mathbf{i}}\right) \sum_{\mathbf{M}} \int d \beta e^{i \mathbf{M}(\mathbf{J}-\mu \pi / 2)} \mathcal{R}_{v}^{\left|M_{v}\right|} \\
& \times|\mathcal{Y}(\theta(E, \beta))|^{2} .
\end{aligned}
$$

This formula can be summed analytically over $M_{v}$, to give the formula

$$
\begin{aligned}
D f(E)= & 2^{7} \pi^{5}\left(E-E_{\mathbf{i}}\right) \sum_{n_{u}=0}^{n_{u}^{\max }} \frac{\left|\mathcal{Y}\left(\theta\left(\beta\left(n_{u} ; E\right)\right)\right)\right|^{2}}{\partial J_{u} / \partial \beta} \\
& \times \Delta\left(J_{v}\left(E, \beta\left(n_{u} ; E\right)\right)-\pi, \lambda_{v}\left(E, \beta\left(n_{u} ; E\right)\right)\right) .
\end{aligned}
$$

We call this the semiquantal formula for the oscillatorstrength density. It has the following meaning.

(1) $\Delta(x, \lambda)$ is a sum of broadened delta functions

$$
\Delta(x, \lambda)=\frac{1}{2 \pi} \frac{\operatorname{coth} \lambda}{1+\left(\frac{\sin (x / 2)}{\sinh \lambda}\right)^{2}} .
$$

When $\lambda \rightarrow 0$, the function $\Delta(x, \lambda)$ tends to $\Sigma_{n=-\infty}^{+\infty} \delta(x$ $-2 \pi n)$. The width of these functions is the natural $\ln$ of the reflection coefficient $\mathcal{R}_{v}$ :

$$
\lambda=\lambda_{v}=-\ln \mathcal{R}_{v}=-\ln \left(1+e^{-2 K(E, \beta)}\right)^{-1 / 2} .
$$

If we take into account the total dependence of the argument $x=J_{v}\left(E, \beta\left(n_{u} ; E\right)\right)-\pi$ on the energy, we come to the following formula for the absorption peak width:

$$
\Gamma=\frac{\frac{\partial J_{u}}{\partial \beta}}{\frac{\partial\left(J_{u}, J_{v}\right)}{\partial(E, \beta)}} \ln \left(1+e^{-2 K}\right) .
$$

When the reflection coefficient is close to 1 (far below the barrier, when $K$ is large and positive), then the $\Delta$ function is narrow and the width is exponentially small:

$$
\Gamma=\frac{\frac{\partial J_{u}}{\partial \beta}}{\frac{\partial\left(J_{u}, J_{v}\right)}{\partial(E, \beta)}} e^{-2 K} .
$$

This formula was initially reported in [13]. When the reflection coefficient is close to zero (far above the barrier, when $K$ is large and negative), the width is large and proportional to K: 


$$
\Gamma=\frac{2 \frac{\partial J_{u}}{\partial \beta}}{\frac{\partial\left(J_{u}, J_{v}\right)}{\partial(E, \beta)}}|K| .
$$

(2) $\beta\left(n_{u} ; E\right)$ is obtained from the quantization condition for $u$ motion, (3.1a):

$$
J_{u}\left(E, \beta\left(n_{u} ; E\right)\right)=\left(n_{u}+1 / 2\right) 2 \pi,
$$

and $\theta\left(\beta\left(n_{u} ; E\right)\right)=\cos ^{-1} \beta\left(n_{u} ; E\right)$ represents the corresponding polar angle, or initial direction of motion of the electron.

(3) For each $E$, since $-1 \leqslant \beta \leqslant 1$, there is a range of integers $n_{u}$ satisfying the $u$-quantization condition (4.13). This gives the limits of summation $0 \leqslant n_{u} \leqslant n_{u}^{\max }$ with $n_{u}^{\max }$ equal to the integer part of $\left[J_{u}(E, 1) / 2 \pi-1 / 2\right]$. Associated with each such integer is a peak of width $\Gamma$, weighted by the angular distribution of outgoing waves at the corresponding angle, times $\left[\partial J_{u} / \partial \beta\right]^{-1}$. The peaks are positioned at energies given by the $v$-quantization condition (3.1b)

$$
J_{v}\left[E, \beta\left(n_{u} ; E\right)\right]+\delta=\left(n_{v}+1 / 2\right) 2 \pi
$$

(in good approximation, we may neglect the imaginary addend $i \lambda_{v}$ when finding the eigenenergies).

This semiquantal formula (4.7)-(4.13) is easily implemented. It gives the result shown as a dotted curve in Fig. 4 [14]. We see that this formula includes the nearly constant continuum, the narrow below-barrier resonances, and the broad above-barrier resonances.

\section{CLOSED-ORBIT SEMICLASSICAL CALCULATION OF THE ABSORPTION SPECTRUM AND THE RECURRENCE SPECTRUM}

We call the above approach "semiquantal" because it uses the WKB approximation, but everything in the theory involves quantum concepts: individual resonance states and lifetimes. Now we present a semiclassical calculation, which uses closed-orbit theory to compute the absorption and recurrence spectra. We shall show that an important feature of the spectrum is associated with classical orbits that undergo nonclassical above-barrier reflection.

The propagation of a quantum wave from the atom toward the dynamical barrier is described in semiclassical approximation by a system of rays_classical orbits obeying Newton's laws. Near the top of the dynamical barrier the semiclassical approximation breaks down and the ray description is no longer valid. The quantum solution near the barrier top yields a transmitted wave and a reflected wave. The transmitted wave corresponds to ordinary Newtonian trajectories that are launched in the escape sector and go to infinity (Fig. 3). The reflected wave can also be correlated with Newtonian trajectories, but the reflection itself is a quantum process that cannot be described by Newtonian mechanics.

For the Stark system, Eq. (1.1), the dynamical barrier lies on a parabolic curve $v=\sqrt{|E| / F}$, which is indicated as the dotted-dashed line in Fig. 5. Examples of closed orbits asso-
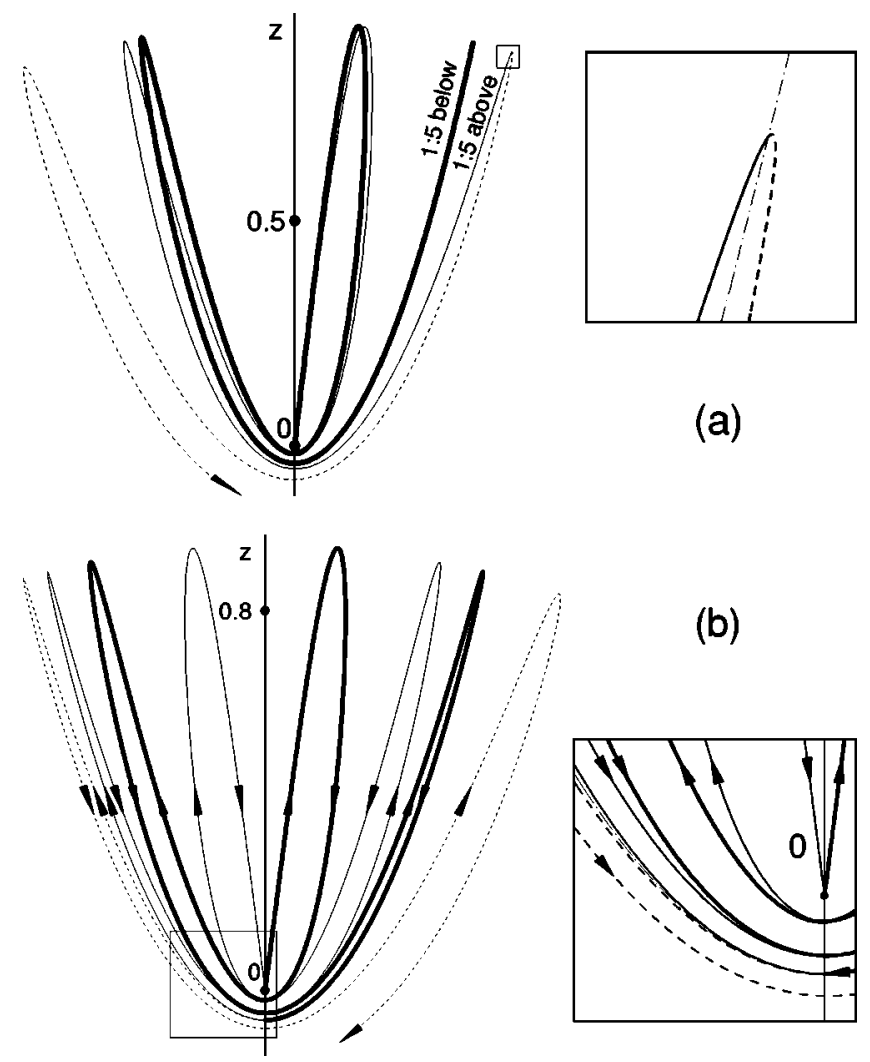

FIG. 5. Two examples of above-barrier-reflected orbits. (a) Heavy line: a below-barrier closed orbit which makes one cycle of $u$ motion and 5 cycles of $v$ motion (1/5 orbit). Light line: abovebarrier-reflected orbit with the same ratio of periods. Classically, an electron would follow the dashed line and escape. (The critical angle $\theta_{c}$ lies between the heavy and light lines.) On the belowbarrier orbit, the electron makes a full stop and then retraces itself. Near the stop-point the trajectory is rectilinear and parallel to the stopping force (the sum of the Coulomb force and the force from the external field). The above-barrier closed orbit also retraces itself. However, at the endpoint, the trajectory does not have a stopping point-the reflection occurs with nonzero velocity. (b) The 1:6 above-barrier closed trajectory shows an example of a "ray splitting" due to above-barrier reflection. The heavy line is the outgoing trajectory; on its third passage through the $-z$ axis, a ray splitting occurs, and the transmitted (classical) trajectory continues to infinity (dashed line) while the above-barrier-reflected trajectory returns to the atom, but not retracing itself (thin line). In the blowups (right) we have indicated the top of the $v$-barrier by the dotted-dashed curve.

ciated with above-barrier reflection are shown. These new nonclassical orbits that arise from above-barrier reflection must be included in closed-orbit theory. (This conclusion is complementary to conclusions reached by Maitra and Heller [15]. They examined the quantum propagator in time- and energy-domains for one-dimensional motion with a potential barrier. One might anticipate that the Green function in the energy domain is a Fourier transform of the time propagator calculated using classical trajectories joining two given points in space. Maitra and Heller show that classically allowed trajectories are not enough; tunneling trajectories must also be considered for energies below the barrier top. Our 
result shows that above the top, nonclassically reflected trajectories make their contribution.)

A quantitative description can be obtained starting again from Eq. (4.6). First let us set the reflection coefficient $\mathcal{R}_{v}$ $=1$, so Eq. (4.6) is equivalent to Eq. (4.4). In that case, it can be shown [11] that if the integral over $\beta$ is evaluated using the stationary-phase approximation, the result is the closedorbit formula for the oscillator-strength-density. Each closed orbit for this regular system is labeled by a pair of integers $\mathbf{M}=\left(M_{u}, M_{v}\right)$ representing the number of cycles of $u$ and $v$ motion before closure. The closed orbit lies on a rational torus characterized by conserved quantities $\left(E, \beta_{\mathbf{M}}\right)$, and the action integral around the closed orbit is

$$
S_{\mathbf{M}}=M_{u} J_{u}\left(E, \beta_{\mathbf{M}}\right)+M_{v} J_{v}\left(E, \beta_{\mathbf{M}}\right) .
$$

The contribution of each closed orbit to the oscillatorstrength-density is

$$
D f_{\mathbf{M}}(E)=\left(\frac{2 \pi}{\partial^{2} S_{\mathbf{M}} / \partial \beta^{2}}\right)^{1 / 2}\left|\mathcal{Y}\left(\theta\left(E, \beta_{\mathbf{M}}\right)\right)\right|^{2} \operatorname{Im} e^{i\left(S_{\mathbf{M}^{-}} \pi / 4\right)} .
$$

Now let us incorporate the effects of tunneling or escape in the $v$ coordinate. Not surprisingly, the effect is that the RHS of Eq. (5.2) is multiplied by the reflection coefficient $\mathcal{R}_{v}^{M_{v}}$ where $M_{v}$ is the number of cycles of $v$ motion before closure. If the reflection coefficient is 1 (impenetrable barrier), we get the standard closed-orbit formula. For a real barrier, $\mathcal{R}_{v}<1$, so the contribution of each closed-orbit is reduced. (The minimal value of $\mathcal{R}_{v}$ for bound motion is reached at the top of the effective potential barrier, where $\mathcal{R}_{v}=2^{-1 / 2}$.) Above the barrier, $\mathcal{R}_{v}$ decreases exponentially.

The resulting formula

$D f_{\mathbf{M}}(E)=\mathcal{R}_{v}^{M_{v}}\left(\frac{2 \pi}{\partial^{2} S_{\mathbf{M}} / \partial \beta^{2}}\right)^{1 / 2}\left|\mathcal{Y}\left(\theta\left(E, \beta_{\mathbf{M}}\right)\right)\right|^{2} \operatorname{Im} e^{i\left(S_{\mathbf{M}}-\pi / 4\right)}$

also applies to nonclassical orbits associated with abovebarrier reflection. In that case the quasiclassical orbit goes out to a complex turning point, and the associated wave is partially reflected. In the present case, we find reflection coefficients of about 0.57 for the above-barrier $1 / 5$ orbit, and about 0.67 for the above-barrier $1 / 6$ orbit.

More generally, Eq. (5.3) is accurate if the closed orbit is well-separated from the endpoints $\beta= \pm 1$, and from the critical point $\beta_{c}$, where the reflection coefficient changes rapidly as a function of $\beta$. (Refinements of a familiar type are necessary otherwise [12].) Such calculations have been carried out, and they give good agreement with semiquantal calculations.

The effect of the classically forbidden above-barrierreflected orbits is dramatically revealed in a recurrence spectrum. The recurrence spectrum is given by $|R(s)|^{2}$ where $R(s)$ is the Fourier transform of $D f(w)$ over a selected range of $w(133.2 \leqslant w \leqslant 138.5$ in our case),

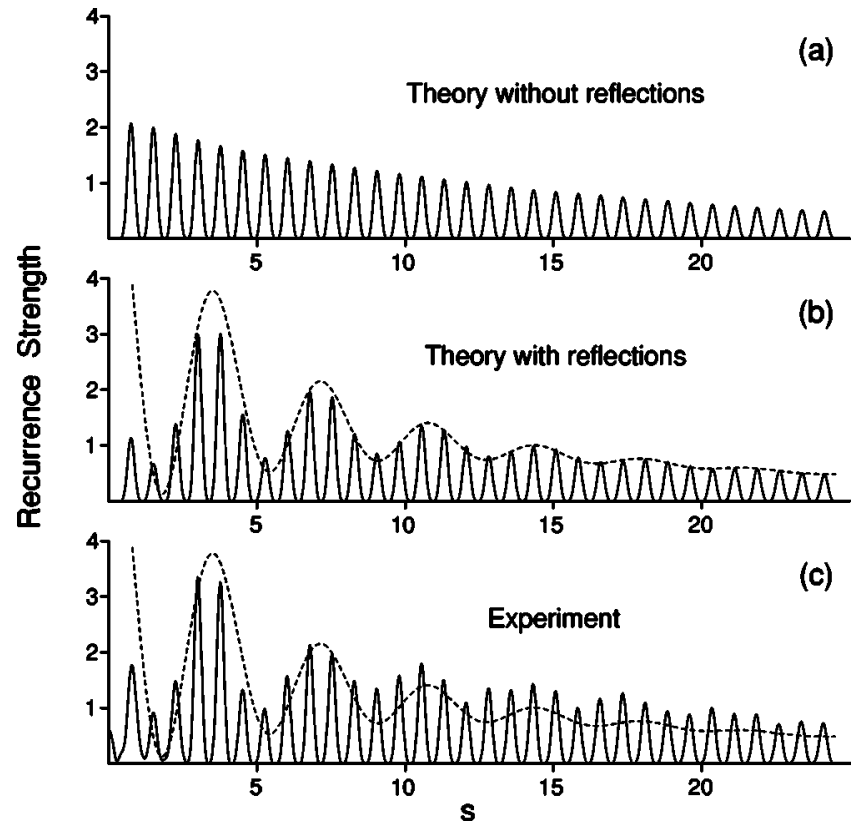

FIG. 6. Recurrence spectra vs the scaled action $s$. The $n$th peak represents the effect of the $n$th return of the parallel orbit combined with all other orbits which have $n$ oscillations of $u$ motion. Dashed line is the theoretical envelope of the modulations, induced by the interference of quasiclassical closed orbits undergoing abovebarrier reflections with the ordinary closed orbits.

$$
R(s)=\int e^{-2 \pi i s w} D f(w) d w .
$$

Closed-orbit theory [2] shows that a recurrence spectrum consists of peaks positioned at the scaled actions $s$ of closed orbits. The recurrence spectrum of the data in Fig. 4 is shown in Figs. 6(b) and 6(c). Large-scale modulations are conspicuous. We shall show that they arise from above-barrier reflections.

For scaled energy $\varepsilon=-0.125$, the action of every orbit is close to a multiple of the action of the parallel orbit $s_{\|}$. Therefore individual orbits are not resolved in Fig. 6: each peak corresponds to all orbits having the same number of oscillations of $u$ motion. If we neglect above-barrier reflections, we get a smoothly decaying sequence of peaks [Fig. 6(a)]. The decay rate is determined by the width of the sharp below-barrier peaks (see Fig. 4); this width is governed by the energy resolution in the experiment.

When above-barrier reflections are taken into account, modulations occur in the recurrence spectrum. The effect is surprisingly large - the modulations are comparable to the peak heights in Fig. 6. We may contrast this with the ratio of the peak heights in the photoexcitation spectrum, Fig. 4, where peak heights associated with above-barrier resonances are only $10 \%$ of the heights of the below-barrier resonances. The enhanced effect of above-barrier reflections in the recurrence spectrum arises because the latter is sensitive to the integrated oscillator-strength $f_{n_{v}}$, which is still considerable for the above-barrier resonances.

In the scaled spectrum, the narrow resonances with $n_{v}$ $=0$ are nearly equally spaced by $2 \pi / s_{\|}[16], s_{\|}$being the 
distance between peaks in the recurrence spectrum. The broad levels are displaced from these by $T_{u} / T_{v}$ fraction of their spacing. $T_{u}$ and $T_{v}$ are the periods of classical motion taken at the values of $E$ and $\beta$ corresponding to the abovebarrier resonance. Taking also into account the resonance widths $\Gamma_{n_{v}}$, we can make the Fourier transform analytically and find the envelope of recurrence peaks:

$$
\begin{aligned}
f(s)= & f_{0}^{2} e^{-2 \pi \Gamma_{0} s}+f_{1}^{2} e^{-2 \pi \Gamma_{1} s} \\
& +2 f_{0} f_{1} e^{-\pi\left(\Gamma_{0}+\Gamma_{1}\right) s} \cos \left(2 \pi \frac{T_{u}}{T_{v}} \frac{s}{s_{\|}}\right) .
\end{aligned}
$$

This envelope is in good agreement with more detailed calculations [Fig. 6(b)]. There is also good agreement in the depth and period of modulation with the experimental recurrence spectrum [Fig. 6(c)].

The closed-orbit sum reveals that the large modulations in the recurrence spectrum result from the interference of classical, below-barrier orbits and nonclassical, above-barrier closed-orbits. The major closed-orbit contributions come from $T_{u} / T_{v}=1 / 4$ and $1 / 5$ below-barrier orbits and from $1 / 5$ and $1 / 6$ above-barrier orbits. The period of modulation of the recurrence spectrum is, according to Eq. (5.5), $T_{u} / T_{v}$. At $E$ and $\beta$ of the broad resonance, $T_{u} / T_{v}=1 / 4.7$, or 4.7 peaks per period.

The peaks at greater scaled actions correspond to longer orbits; many of them are repetitions of the shorter ones. The decay in the modulation amplitude arises because the recurrence strengths of above-barrier orbits decrease as a power of the reflection coefficient.

\section{CONCLUSION}

We have measured the scaled photoabsorption spectrum of the Li atom in an external electric field. It shows a periodic sequence of sharp peaks associated with quasibound states, and it shows broad peaks, identified as classically forbidden above-barrier resonances. These broad low-amplitude peaks have a dramatic effect on the recurrence spectrum, introducing about $100 \%$ modulation. We developed a semiclassical theory of the oscillator-strength-density that is in agreement with experimental data. We have shown that the modulation carries important information about abovebarrier resonances: the depth of modulation is related with the integrated oscillator-strength of the resonances, and the decay rate is determined by the width of resonances. The closed-orbit interpretation of the effect involves nonNewtonian orbits that undergo above-barrier reflection.

\section{ACKNOWLEDGMENTS}

This research was supported by the National Science Foundation and the Office of Naval Research.
[1] E. C. Kemble, The Fundamental Principles of Quantum Mechanics with Elementary Applications (Dover, New York, 1958), Chap. III, Sec. 21j.

[2] M. L. Du and J. B. Delos, Phys. Rev. A 38, 1896 (1988); 38, 1913 (1988).

[3] H. C. Bryant et al., Phys. Rev. Lett. 58, 2412 (1987).

[4] R. R. Freeman and N. P. Economou, Phys. Rev. A 20, 2356 (1979); E. Luc-Koenig and A. Bachelier, Phys. Rev. Lett. 43, 921 (1979).

[5] A. D. Peters, C. Jaffé, and J. B. Delos, Phys. Rev. A 56, 331 (1997), and references therein.

[6] H. Rottke and K. H. Welge, Phys. Rev. A 33, 301 (1986).

[7] Using hindsight, we can see evidence of peaks possibly associated with classically forbidden above-barrier reflections in the following, though the phenomenon was not addressed: W. Sandner, K. A. Safinya, and T. F. Gallagher, Phys. Rev. A 23, 2448 (1981); T. S. Luk, L. DiMauro, T. Bergeman, and H. Metcalf, Phys. Rev. Lett. 47, 83 (1981); H. Rottke and K. H. Welge, Phys. Rev. A 33, 301 (1986); W. L. Glab, K. Ng, D. Yao, and M. H. Nayfeh, ibid. 31, 3677 (1985); 35, 2508 (1987). Other studies examine a similar range of parameters, but do not provide evidence of classically forbidden processes: G. M. Lankhuijzen and L. D. Noordam, Phys. Rev. Lett. 76, 1784 (1996); F. Robicheaux and J. Shaw, ibid. 77, 4154 (1996), Phys. Rev. A 56, 278 (1997).
[8] (a) U. Eichmann, K. Richter, D. Wintgen, and W. Sandner, Phys. Rev. Lett. 61, 2438 (1988); (b) M. Courtney, N. Spellmeyer, H. Jiao, and D. Kleppner, Phys. Rev. A 51, 3604 (1995).

[9] M. S. Child, Semiclassical Mechanics with Molecular Applications (Oxford University Press, New York, 1991). Our complex quantization conditions (3.1) can be obtained with help of connection formulas discussed in Chap. 3, Secs. 3.2 and 3.5.

[10] V. Kondratovich and J. B. Delos, Phys. Rev. A 56, R5 (1997); 57, 4654 (1998).

[11] M. W. Beims, V. Kondratovich, and J. B. Delos, Phys. Rev. A 62, 043401 (2000).

[12] M. V. Berry and M. Tabor, Proc. R. Soc. London, Ser. A 349, 101 (1976). A valuable addition to this work can be found in the paper by P. J. Richens, J. Phys. A 15, 2101 (1982).

[13] M. W. Beims, V. Kondratovich, and J. B. Delos, Phys. Rev. Lett. 81, 4537 (1998).

[14] Actually the quasidiscrete peaks with $n_{2}=0$ are narrower and higher than indicated in Fig. 3. We artificially broadened them in a manner consistent with the experimental resolution.

[15] N. T. Maitra and E. J. Heller, in Classical, Semiclassical and Quantum Dynamics in Atoms, edited by H. Friedrich and B. Eckhardt (Springer, New York, 1997).

[16] V. Kondratovich and J. B. Delos, Phys. Rev. A 57, 4604 (1998). 